\title{
AN INTERIOR INVERSE PROBLEM FOR THE IMPULSIVE DIRAC OPERATOR
}

\author{
A. S. OZKAN AND R. KH. AMIROV
}

Abstract. In this study, an inverse problem for Dirac differential operators with discontinuities is studied. It is shown that the potential function can be uniquely determined by a set of values of eigenfunctions at some internal point and one spectrum.

\section{Introduction}

Let us consider the system of Dirac differential equations

$$
\ell[y(x)]:=B y^{\prime}(x)+\Omega(x) y(x)=\lambda y(x), \quad x \in I:=\left(0, \frac{\pi}{2}\right) \cup\left(\frac{\pi}{2}, \pi\right)
$$

where $B=\left(\begin{array}{cc}0 & 1 \\ -1 & 0\end{array}\right), \Omega(x)=\left(\begin{array}{cc}p(x) & q(x) \\ q(x) & -p(x)\end{array}\right), y(x)=\left(\begin{array}{l}y_{1}(x) \\ y_{2}(x)\end{array}\right), p(x)$ and $q(x)$ are real valued functions in $L_{2}(0, \pi), \lambda$ is spectral parameter.

We denote by $L$ the boundary value problem generated by Equation (1.1) with the boundary and discontinuity conditions

$$
\begin{aligned}
& U(y):=y_{1}(0)=0 \\
& V(y):=y_{2}(\pi)=0 \\
& C(y):=y\left(\frac{\pi}{2}+0\right)=A y\left(\frac{\pi}{2}-0\right)
\end{aligned}
$$

where $A=\left(\begin{array}{cc}\beta & 0 \\ 0 & \beta^{-1}\end{array}\right), \beta \in \mathbb{R}^{+} \backslash\{1\}$.

The basic and comprehensive results about Dirac operators were given in [7]. Furthermore, spectral problems for Sturm-Liouville or Dirac operators extensively well studied in [2], [3] and [4].

Corresponding author: R. Kh. Amirov. 2000 Mathematics Subject Classification. 34A55, 34B24, 34L05.

Key words and phrases. Dirac operator, determination of the potential, discontinuous condition, interior spectral data. 
Boundary value problems with discontinuities inside the interval often appear in mathematics, mechanics, physics, geophysics and other branches of natural properties. The jump conditions like (1.4) appear in some important physical problem. [5] is well known work about discontinious inverse eigenvalue problems. Direct and inverse problems for Dirac operators with discontinuities inside the interval were investigated in [1]. Inverse problems for interior spectral data of the Sturm-Liouville or Dirac operators were studied in [8], [9] and [10]

In the present paper, we consider a Dirac operator with jump conditions inside interval. We prove by using the similar methods in [6] and [9], that the potential function $q(x)$ can be uniquely determined by a set of values of eigenfunctions and sequence of eigenvalues.

\section{Preliminaries}

Let the functions $\varphi(\cdot, \lambda): I \rightarrow R^{2}$ be solution of equation (1.1) satisfy the initial conditions:

$$
\varphi(0, \lambda)=\left(\begin{array}{c}
0 \\
-1
\end{array}\right)
$$

and the jump conditions (1.4).

It is shown in [1] that, the solution $\varphi(x, \lambda)$ has a representation as follows;

$$
\varphi(x, \lambda)=\varphi_{0}(x, \lambda)+\int_{0}^{x} K(x, t)\left(\begin{array}{c}
\sin \lambda t \\
-\cos \lambda t
\end{array}\right) d t
$$

where $\varphi_{0}(x, \lambda)=\left(\varphi_{01}(x, \lambda), \varphi_{02}(x, \lambda)\right)^{T}$ has the form

$$
\begin{gathered}
\varphi_{01}(x, \lambda)=\left\{\begin{array}{cc}
\sin \lambda x, & 0<x<\frac{\pi}{2}, \\
\beta^{+} \cos \lambda x+\beta^{-} \cos \lambda(\pi-x), & \frac{\pi}{2}<x<\pi,
\end{array}\right. \\
\varphi_{02}(x, \lambda)=\left\{\begin{array}{ll}
-\cos \lambda x, & 0<x<\frac{\pi}{2}, \\
\beta^{+} \sin \lambda x-\beta^{-} \sin \lambda(\pi-x), & \frac{\pi}{2}<x<\pi,
\end{array},\right.
\end{gathered}
$$

$\beta^{ \pm}=\frac{1}{2}\left(\beta \pm \beta^{-1}\right), K(x, t)=\left(K_{i j}(x, t)\right)_{i, j=1,2}, K_{i j}(x,.) \in L_{2}(-x, x)$ for $i, j=1,2$ and every fixed $x$.

The function $\Delta(\lambda)$ which has the form:

$$
\Delta(\lambda)=\varphi_{2}(\pi, \lambda)
$$

is the characteristic function of the problem (1.1)-(1.4), i.e. it's zeros are precisely eigenvalues.

From the equality (2.2)-(2.5) we have:

$$
\Delta(\lambda)=\beta^{+} \sin \lambda \pi+o(\exp \tau \pi)
$$

where, $\tau=|\operatorname{Im} \lambda|$. The following lemma can be proved by similar methods in [1]. 
Lemma 1. (i) The problem L has countable many eigenvalues, such that all of them are real and simple.

(ii) The eigenvalues $\lambda_{n}$ are given by the following asymptotic formulae:

$$
\lambda_{n}=n+O\left(\frac{1}{n}\right)
$$

\section{Main result}

Together with $\widetilde{L}$, we consider the problem $L(\widetilde{\Omega})$ of the same form but with a different coefficient $\Omega$ such that

$$
\widetilde{\Omega}(x)=\left(\begin{array}{cc}
\widetilde{p}(x) & q(x) \\
q(x) & -\widetilde{p}(x)
\end{array}\right)
$$

It is assumed in what follows that if a certain symbol $s$ denotes an object related to $L$, then the corresponding symbol $\widetilde{s}$ with tilde denote the analogous object related to $\widetilde{L}$. The eigenvalues and the corresponding eigenfunctions of the problem $L$ are denoted by $\lambda_{n}$ and $\varphi_{n}(x)=$ $\varphi\left(x, \lambda_{n}\right)$, respectively.

Now we state the main result of this work. Denote $W[y, z](x):=y_{1}(x) z_{2}(x)-y_{2}(x) z_{1}(x)$ for $y=\left(y_{1}, y_{2}\right)^{T}$ and $z=\left(z_{1}, z_{2}\right)^{T}$

Theorem 1. If $\lambda_{n}=\widetilde{\lambda}_{n}$ and $W\left[\varphi_{n}, \widetilde{\varphi}_{n}\right]\left(\frac{\pi}{2}-0\right)=0$ for all $n \in \mathbb{N}$, then $p(x)=\widetilde{p}(x)$, a.e. in $[0, \pi]$.

Proof. Let us write the equation (1.1) for the solutions $\varphi$ and $\widetilde{\varphi}$

$$
\begin{aligned}
& B \varphi^{\prime}(x, \lambda)+\Omega(x) \varphi(x, \lambda)=\lambda \varphi(x, \lambda) \\
& B \widetilde{\varphi}^{\prime}(x, \lambda)+\widetilde{\Omega}(x) \varphi(x, \lambda)=\lambda \widetilde{\varphi}(x, \lambda)
\end{aligned}
$$

If we multiply these equalities by $\widetilde{\varphi}(x, \lambda)$ and $\varphi(x, \lambda)$ respectively and subtract then we get

$$
\frac{d}{d x}\left\{\varphi_{1}(x, \lambda) \widetilde{\varphi}_{2}(x, \lambda)-\widetilde{\varphi}_{1}(x, \lambda) \varphi_{2}(x, \lambda)\right\}=[\Omega(x)-\widetilde{\Omega}(x)] \varphi(x, \lambda) \widetilde{\varphi}(x, \lambda)
$$

Integrating last equality from 0 to $\frac{\pi}{2}$ with respect to $x$, the equality

$$
\begin{aligned}
\left.\left\{\varphi_{1}(x, \lambda) \widetilde{\varphi}_{2}(x, \lambda)-\widetilde{\varphi}_{1}(x, \lambda) \varphi_{2}(x, \lambda)\right\}\right|_{0} ^{\frac{\pi}{2}} & =\int_{0}^{\frac{\pi}{2}}[\Omega(x)-\widetilde{\Omega}(x)] \varphi(x, \lambda) \widetilde{\varphi}(x, \lambda) d x \\
& =\int_{0}^{\frac{\pi}{2}}[p(x)-\widetilde{p}(x)] J \varphi(x, \lambda) \widetilde{\varphi}(x, \lambda) d x
\end{aligned}
$$

is obtained, where $J:=\left(\begin{array}{cc}1 & 0 \\ 0 & -1\end{array}\right)$. If we denote

$$
F(\lambda):=\int_{0}^{\frac{\pi}{2}}[p(x)-\widetilde{p}(x)] J \varphi(x, \lambda) \widetilde{\varphi}(x, \lambda) d x
$$


then we obtain from the initial condition (2.1) and the assumption $W\left[\varphi_{n}, \widetilde{\varphi}_{n}\right]\left(\frac{\pi}{2}-0\right)=0$ in hypothesis that

$$
F\left(\lambda_{n}\right)=
$$

for all $n$.

Now, define $\chi(\lambda):=\frac{F(\lambda)}{\Delta(\lambda)}$ which is entire function from above argument. Since $F(\lambda)=$ $O(\exp \tau \pi)$ and $|\Delta(\lambda)| \geq C \exp \tau \pi$ for $\lambda \in G_{\epsilon}:=\left\{\lambda:\left|\lambda-\lambda_{n}\right|>\varepsilon\right\}$ then $\chi(\lambda)$ is constant from the Liouville's theorem. Moreover,

$$
\lim _{\substack{\lambda \rightarrow \infty \\ \lambda \in \mathbb{R}}} \chi(\lambda)=0
$$

from the equality (2.2)-(2.4) and Riemann-Lebesque lemma. Therefore $\chi(\lambda)=0$ on the whole $\lambda$-plane. On the other hand, one can easily obtaine that

$$
J \varphi(x, \lambda) \widetilde{\varphi}(x, \lambda)=-\cos 2 \lambda x+\int_{0}^{x} K_{1}(x, t) \cos 2 \lambda t d t+\int_{0}^{x} K_{2}(x, t) \sin 2 \lambda t d t
$$

where $K_{i}(x, t), i=1,2$, depend only on $x, t$. It follows from (3.5) and (3.7) that

$$
\int_{0}^{\frac{\pi}{2}} P(x)\left\{\cos 2 \lambda x-\int_{0}^{x} K_{1}(x, t) \cos 2 \lambda t d t-\int_{0}^{x} K_{2}(x, t) \sin 2 \lambda t d t\right\} d x=0
$$

for all $\lambda$, where $P(x):=[p(x)-\widetilde{p}(x)]$. This can be rewritten as

$$
\begin{array}{r}
\int_{0}^{\frac{\pi}{2}} \cos 2 \lambda \tau\left[P(\tau)+\int_{\tau}^{\frac{\pi}{2}} P(x) K_{1}(x, t) d x\right] d t \\
+\int_{0}^{\frac{\pi}{2}} \sin 2 \lambda t \int_{\tau}^{\frac{\pi}{2}} P(x) K_{2}(x, t) d x d t=0
\end{array}
$$

or

$$
\int_{0}^{\frac{\pi}{2}}\left(\begin{array}{c}
P(\tau)+\int_{\tau}^{\frac{\pi}{2}} P(x) K_{1}(x, t) d x \\
\int_{\tau}^{\frac{\pi}{2}} P(x) K_{2}(x, t) d x
\end{array}\right)(\cos 2 \lambda \tau, \sin 2 \lambda t)^{T} d t=0
$$

Therefore, it follows from the completeness of the vector functions $(\cos 2 \lambda \tau, \sin 2 \lambda t)^{T}$ in $L_{2}(0, \pi) \oplus$ $L_{2}(0, \pi)$ that $P(x)=0$, i.e. $p(x)=\widetilde{p}(x)$ for $x \in\left(0, \frac{\pi}{2}\right)$.

To show that $p(x)=\widetilde{p}(x)$ for $x \in\left(\frac{\pi}{2}, \pi\right)$ we should repeat arguments for the supplementary problem

$$
\begin{aligned}
B y^{\prime}(x)+\Omega(\pi-x) y(x) & =\lambda y(x) \quad x \in I \\
y_{1}(0) & =0 \\
y_{2}(\pi) & =0 \\
y\left(\frac{\pi}{2}-0\right) & =A y\left(\frac{\pi}{2}+0\right) .
\end{aligned}
$$

Since $p(\pi-x)=\widetilde{p}(\pi-x)$ for $x \in\left(0, \frac{\pi}{2}\right)$, we obtaine that $p(x)=\widetilde{p}(x)$ for $x \in\left(\frac{\pi}{2}, \pi\right)$. This completes the proof. 


\section{References}

[1] R. Kh. Amirov, On system of Dirac differential equations with discontinuity conditions inside an interval, Ukrainian Math. J., 57(2005), 712-727.

[2] G. Freiling and V. A. Yurko, Inverse Sturm-Liouville Problems and Their Applications, Huntington, NY: Nova Science, 2001

[3] M. G. Gasymov and B. M. Levitan, The inverse problem for a dirac system, Dokl. Akad. Nauk SSSR, 167 (1966), 967-970.

[4] I. M. Gelfand and B. M. Levitan, On the determination of a differential equation from its spectral function, Amer. Math. Transl., 1 (1951), 239-253.

[5] O. H. Hald, Discontiuous inverse eigenvalue problems, Comm. Pure Appl. Math., 37(1984), 539-577.

[6] H. Hochstadt and B. Lieberman, An inverse Sturm-Liouville problem with mixed given data, SIAM J. Appl. Math., 34 (1978), 676-680.

[7] B. M. Levitan, and I. S. Sargsyan, Sturm-Liouville and Dirac Operators Kluwer Academic Publishers, Dodrecht/Boston/London, 1991.

[8] K. Mochizuki and I. Trooshin, Inverse problem for interior spectral data of Sturm Liouville operator, J. Inverse Ill-posed Probl., 9 (2001), 425-433.

[9] K. Mochizuki and I. Trooshin, Inverse problem for interior spectral data of the Dirac operator on a finite interval, Publ. RIMS, Kyoto Univ., 38 (2002), 387-395.

[10] Chuan-Fu Yang and Xiao-Ping Yang, An interior inverse problem for the Sturm Liouville operator with discontinuous conditions, Applied Mathematics Letters, 22 (2009), 1315-1319.

Department of Mathematics Faculty of Art\&Sci. Cumhuriyet University 58140 Sivas, Turkey.

E-mail: sozkan@cumhuriyet.edu.tr

Department of Mathematics Faculty of Art\&Sci. Cumhuriyet University 58140 Sivas, Turkey.

E-mail: emirov@cumhuriyet.edu.tr 